\title{
IDENTITIES FOR $q$-ULTRASPHERICAL POLYNOMIALS AND JACOBI FUNCTIONS
}

\author{
H. T. KOELINK
}

(Communicated by Hal L. Smith)

\begin{abstract}
A $q$-analogue of a result by Badertscher and Koornwinder [Canad. J. Math. 44 (1992), 750-773] relating the action of a Hahn polynomial of differential operator argument on ultraspherical polynomials to an ultraspherical polynomial of shifted order and degree is derived. The $q$-analogue involves $q$-Hahn polynomials, continuous $q$-ultraspherical polynomials, and a shift operator. Another limit as $q$ tends to 1 yields an identity for Jacobi functions. Combination with another result of Badertscher and Koornwinder gives a curious formula for Jacobi functions.
\end{abstract}

\section{INTRODUCTION}

Badertscher and Koornwinder [4] have proved a formula in which a Hahn polynomial of differential operator argument acts on ultraspherical polynomials. Explicitly, this formula is (cf. [4, Corollary 6.3])

$Q_{k}\left(\frac{1}{2} l-\frac{i}{2} \frac{d}{d \theta} ; \lambda-1, \lambda-1, l\right) C_{l}^{\lambda}(\cos \theta)=\frac{(\lambda)_{k}}{(l-k+1)_{k}}(2 i)^{k}(\sin \theta)^{k} C_{l-k}^{\lambda+k}(\cos \theta)$

for $k, l \in \mathbf{Z}_{+}, 0 \leq k \leq l, \lambda \notin-\frac{1}{2} \mathbf{Z}_{+}$. In (1.1) $C_{l}^{\lambda}$ denotes an ultraspherical polynomial,

$$
C_{l}^{\lambda}(\cos \theta)=\sum_{n=0}^{l} \frac{(\lambda)_{n}(\lambda)_{l-n}}{n !(l-n) !} e^{i(l-2 n) \theta},
$$

and $Q_{k}$ is a Hahn polynomial,

$$
Q_{k}(x ; a, b, N)={ }_{3} F_{2}\left(\begin{array}{c}
-k, a+b+k+1,-x ; 1 \\
a+1,-N
\end{array}\right),
$$

with $N \in \mathbf{N}, k, x \in\{0, \ldots, N\}$ (cf. the references in [4]). Here we use the

Received by the editors December 21, 1993.

1991 Mathematics Subject Classification. Primary 33D45, 33C45, 33D80.

Key words and phrases. Continuous $q$-ultraspherical polynomial, $q$-Hahn polynomial, Jacobi function, ultraspherical polynomial, continuous symmetric Hahn polynomial.

Supported by a NATO-Science Fellowship of the Netherlands Organization for Scientific Research (NWO). 
standard notation

$$
{ }_{r+1} F_{r}\left(\begin{array}{c}
a_{1}, \ldots, a_{r+1} \\
b_{1}, \ldots, b_{r}
\end{array} ; z\right)=\sum_{k=0}^{\infty} \frac{\left(a_{1}\right)_{k} \ldots\left(a_{r+1}\right)_{k}}{\left(b_{1}\right)_{k} \ldots\left(b_{r}\right)_{k}} \frac{z^{k}}{k !}, \quad(a)_{k}=\prod_{i=0}^{k-1}(a+i)
$$

for hypergeometric series.

Badertscher and Koornwinder [4] (see also [13]) prove (1.1) using harmonic analysis on the sphere $S O(d+1) / S O(d)$. The spherical functions on this homogeneous space are the ultraspherical polynomials $C_{l}^{(d-1) / 2}(\cos \theta)$ and the associated spherical functions are of the form $(\sin \theta)^{k} C_{l-k}^{(d-1) / 2+k}(\cos \theta)$, so (1.1) shows how to obtain the associated spherical functions from the spherical functions in this particular case. The variable $\theta$ denotes the geodesic distance on the sphere to some fixed point.

The continuous $q$-ultraspherical polynomials

$$
C_{l}(\cos \theta ; \beta \mid q)=\sum_{n=0}^{l} \frac{(\beta ; q)_{n}(\beta ; q)_{l-n}}{(q ; q)_{n}(q ; q)_{l-n}} e^{i(l-2 n) \theta}
$$

(cf. Askey and Ismail [1], Askey and Wilson [3, §4], Gasper and Rahman [7, §7.4]) are $q$-analogues of the ultraspherical polynomials (1.2), since $\lim _{q \uparrow 1} C_{l}\left(x ; q^{\lambda} \mid q\right)=C_{l}^{\lambda}(x)$. The $q$-shifted factorials in (1.4) are defined by

$$
(a ; q)_{k}=\prod_{i=0}^{k-1}\left(1-a q^{i}\right), \quad(a ; q)_{\infty}=\lim _{k \rightarrow \infty}(a ; q)_{k} .
$$

Here we follow the notation of the book [7] by Gasper and Rahman. We assume that $q \in(0,1)$.

In case $\beta=q^{\frac{1}{2}}$ the continuous $q$-ultraspherical polynomials, which are $q$ analogues of the Legendre polynomials, have an interpretation as zonal spherical elements on the quantum $S U(2)$ group (cf. Koornwinder [15]). The continuous $q$-ultraspherical polynomials $C_{l-k}\left(x ; q^{\frac{1}{2}+k} \mid q\right)$ have an interpretation as associated spherical elements on the quantum $S U(2)$ group (cf. Koelink [9] and Noumi and Mimachi [17], [18]).

Since the group $S U(2)$ is a double covering of $S O(3)$, we can use similar ideas as used in Badertscher and Koornwinder [4] in the quantum $S U(2)$ group setting to obtain the following identity for $\beta=q^{\frac{1}{2}}$ (cf. $\left.[10, \S 10]\right)$.

Proposition 1.1. For $k, l \in \mathbf{Z}_{+}, 0 \leq k \leq l$, and $\beta \notin q^{-\frac{1}{2} \mathbf{Z}_{+}}$we have the following identity for the continuous q-ultraspherical polynomials defined in (1.4):

$$
\begin{aligned}
& Q_{k}\left(q^{-\frac{1}{2} l} E_{+} ; \beta / q, \beta / q, l ; q\right) C_{l}(\cos \theta ; \beta \mid q) \\
& \quad=\frac{(\beta ; q)_{k}}{\left(q^{l-k+1} ; q\right)_{k}}\left(\beta e^{-2 i \theta} ; q\right)_{k} e^{i k \theta} C_{l-k}\left(\cos \theta ; \beta q^{k} \mid q\right) .
\end{aligned}
$$

Here $Q_{k}$ are $q$-Hahn polynomials defined by

$$
Q_{k}(x ; a, b, N ; q)={ }_{3} \varphi_{2}\left(\begin{array}{c}
q^{-k}, a b q^{k+1}, x \\
a q, q^{-N}
\end{array} ; q, q\right)
$$

and $E_{+}$is the operator defined by $\left(E_{+} f\right)\left(e^{i \theta}\right)=f\left(e^{i \theta} \sqrt{q}\right)$. 
The notation for basic (or $q$-) hypergeometric series in (1.7) is taken from [7, Chapter 1]. The basic hypergeometric series is defined by

$$
{ }_{r+1} \varphi_{r}\left(\begin{array}{c}
a_{1}, \ldots, a_{r+1} \\
b_{1}, \ldots, b_{r}
\end{array} q, z\right)=\sum_{k=0}^{\infty} \frac{\left(a_{1} ; q\right)_{k} \cdots\left(a_{r+1} ; q\right)_{k}}{\left(b_{1} ; q\right)_{k} \cdots\left(b_{r} ; q\right)_{k}} \frac{z^{k}}{(q ; q)_{k}}
$$

where the $q$-shifted factorial is defined in (1.5).

The $q$-Hahn polynomials can be viewed as a special case of the $q$-Racah polynomials (cf. Askey and Wilson [2], Gasper and Rahman [7, §7.2]). The operator $E_{+}$is defined by Askey and Wilson (cf. [3, (5.2)]). Note that the operator $E_{+}$is well defined on polynomials.

As stated before, the original approach to (1.6) in case $\beta=q^{1 / 2}$ is motivated by the representation theory of the quantum $S U(2)$ group. This approach shows that in case $\beta=q^{1 / 2}(1.6)$ can be generalised in the sense that we can replace the continuous $q$-Legendre polynomial by a one-parameter family of Askey-Wilson polynomials (cf. (2.1)). However, the corresponding right-hand side of (1.6) becomes a sum of two functions instead of one (cf. [10, Proposition 10.4]).

It is the purpose of this note to prove (1.6) in full generality by analytic means. This is done in $\S 2$, in which also the limit case $q \uparrow 1$ of (1.6) to (1.1) is presented. In $\S 3$ we present another limit transition of (1.6) as $q \uparrow 1$ to an identity involving Jacobi functions using the (rigorous) limit transition of the continuous $q$-ultraspherical polynomials to the Jacobi functions given by Koornwinder [14]. A simple combination with another result of Badertscher and Koornwinder results in a formula relating derivatives of Jacobi functions with Jacobi functions of shifted degree. For information and references on Jacobi functions the reader may consult the papers [11], [12] by Koornwinder.

\section{PROOF OF THE MAIN RESULT}

In order to prove (1.6) we start with the generating function for the AskeyWilson polynomials. The Askey-Wilson polynomials (cf. [3]) are defined by (2.1)

$$
\begin{aligned}
p_{n}(\cos \theta ; a, b, c, d \mid q)= & a^{-n}(a b ; q)_{n}(a c ; q)_{n}(a d ; q)_{n} \\
& \times{ }_{4} \varphi_{3}\left(\begin{array}{c}
\left.q^{-n}, a b c d q^{n-1}, a e^{i \theta}, a e^{-i \theta} ; q, q\right) . \\
a b, a c, a d
\end{array}\right.
\end{aligned}
$$

Ismail and Wilson proved the following generating function for the AskeyWilson polynomials (cf. [8, (1.9)])

(2.2)

$\sum_{n=0}^{\infty} \frac{p_{n}(y ; a, b, c, d \mid q) t^{n}}{(a b ; q)_{n}(d c ; q)_{n}(q ; q)_{n}}={ }_{2} \varphi_{1}\left(\begin{array}{c}a / z, b / z \\ a b\end{array} ; q, z t\right){ }_{2} \varphi_{1}\left(\begin{array}{c}c z, d z \\ c d\end{array} ; q, t / z\right)$,

where $y=\left(z+z^{-1}\right) / 2$.

In the generating function (2.2) we replace $a, b, c, d, z, t$ by $\sqrt{a b q}, 0$, $\sqrt{a q / b}, q^{-N} / \sqrt{a b q}, q^{x} \sqrt{a b q}, t \sqrt{a q / b}$ for $N \in \mathbf{N}$ and $x \in\{0, \ldots, N\}$. For these choices the Askey-Wilson polynomial reduces to a dual $q$-Hahn poly- 
nomial (cf. $[7, \S 7.2]$ )

$$
\begin{aligned}
p_{n}(y)= & (a b q)^{-n / 2}(a q ; q)_{n}\left(q^{-N} ; q\right)_{n} \\
& \times{ }_{3} \varphi_{2}\left(\begin{array}{c}
\left.q^{-n}, a b q^{x+1}, q^{-x} ; q, q\right), \quad n \leq N, \\
a q, q^{-N}
\end{array}\right.
\end{aligned}
$$

and $p_{n}(y)=0$ for $n>N$. A straightforward calculation and an application of the $q$-binomial theorem, ${ }_{1} \varphi_{0}\left(q^{-x} ;-; q, z\right)=\left(q^{-x} z ; q\right)_{x}, x \in \mathbf{Z}_{+}$(cf. [7, (1.3.14)]) shows that (2.2) transforms into the following generating function for the dual $q$-Hahn polynomials

$$
\begin{array}{r}
\sum_{n=0}^{N} t^{n} \frac{(a q ; q)_{n}(b q ; q)_{N-n}}{(q ; q)_{n}(q ; q)_{N-n}}{ }_{3} \varphi_{2}\left(\begin{array}{c}
q^{-n}, a b q^{x+1}, q^{-x} \\
a q, q^{-N}
\end{array} q, q\right) \\
=\frac{(b q ; q)_{N}}{(q ; q)_{N}}(a q t ; q)_{x} \varphi_{1}\left(\begin{array}{c}
a q^{x+1}, q^{x-N} \\
q^{-N} / b
\end{array} ; q, t q^{-x} / b\right) .
\end{array}
$$

In (2.4) we specialise $a=b=\beta / q, t=e^{-2 i \theta}, N=l, x=k$ and we multiply by $e^{i l \theta}$ to get

$$
\begin{gathered}
\sum_{n=0}^{l}{ }_{3} \varphi_{2}\left(\begin{array}{c}
q^{-n}, \beta^{2} q^{k-1}, q^{-k} \\
\beta, q^{-l}
\end{array} ;, q\right) \frac{(\beta ; q)_{n}(\beta ; q)_{l-n}}{(q ; q)_{n}(q ; q)_{l-n}} e^{i(l-2 n) \theta} \\
=\frac{(\beta ; q)_{l}}{(q ; q)_{l}}\left(\beta e^{-2 i \theta} ; q\right)_{k} e^{i l \theta}{ }_{2} \varphi_{1}\left(\begin{array}{c}
\beta q^{k}, q^{k-l} \\
q^{1-l} / \beta
\end{array} q, e^{-2 i \theta} q^{1-k} / \beta\right) \\
=\frac{(\beta ; q)_{k}}{\left(q^{l-k+1} ; q\right)_{k}}\left(\beta e^{-2 i \theta} ; q\right)_{k} e^{i k \theta} C_{l-k}\left(\cos \theta ; \beta q^{k} \mid q\right)
\end{gathered}
$$

for $k \in\{0, \ldots, N\}$. The last equality follows from the expression of the continuous $q$-ultraspherical polynomial in terms of a ${ }_{2} \varphi_{1}$-series (cf. [1, (3.2)], $[3,(4.4)],[7,(7.4 .2)])$. This argument is perfectly valid for all $\beta \in \mathbf{C}$ which are not of the form $q^{p}$ for $p \in-\frac{1}{2} \mathbf{Z}_{+}$. Since $\left(q^{-(1 / 2) l} E_{+} ; q\right)_{m} e^{i(l-2 n) \theta}=$ $\left(q^{-n} ; q\right)_{m} e^{i(l-2 n) \theta}$, we can interchange the ${ }_{3} \varphi_{2}$-series and the summation in (2.5) if we replace the upper parameter $q^{-n}$ of the ${ }_{3} \varphi_{2}$-series by $q^{-(1 / 2) l} E_{+}$. Finally use (1.7) to finish the proof of (1.6).

In order to show that (1.6) tends to (1.1) as $q \uparrow 1$ we replace $\beta$ by $q^{\lambda}$ in (2.5) and we take the limit $q \uparrow 1$. Note that all sums and products are finite so we can take termwise limits to find

$$
\begin{gathered}
\sum_{n=0}^{l}{ }_{3} F_{2}\left(\begin{array}{c}
-n, 2 \lambda+k-1,-k \\
\lambda,-l
\end{array} ;\right) \frac{(\lambda)_{n}(\lambda)_{l-n}}{n !(l-n) !} e^{i(l-2 n) \theta} \\
=\frac{(\lambda)_{k}}{(l-k+1)_{k}}\left(1-e^{-2 i \theta}\right)^{k} e^{i k \theta} C_{l-k}^{\lambda+k}(\cos \theta) .
\end{gathered}
$$

Now note that $\left(-\frac{1}{2} l+\frac{i}{2} \frac{d}{d \theta}\right) e^{i(l-2 n) \theta}=-n e^{i(l-2 n) \theta}$, so that $\left(-\frac{1}{2} l+\frac{i}{2} \frac{d}{d \theta}\right)_{m} e^{i(l-2 n) \theta}$ $=(-n)_{m} e^{i(l-2 n) \theta}$. We can interchange the summation and the ${ }_{3} F_{2}$-series on the left-hand side of (2.6) if we replace $-n$ as upper parameter by $-\frac{1}{2} l+\frac{i}{2} \frac{d}{d \theta}$. This result can easily be rewritten as (1.1) by use of (1.2) and (1.3).

Equation (2.6) gives another interpretation of the result (1.1) of Koornwinder and Badertscher as a generating function for the Hahn polynomial 
$Q_{n}(k ; \lambda-1, \lambda-1, l)$. Note that the roles of argument and degree are interchanged in the Hahn polynomial.

\section{LIMIT RELATION FOR JACOBI FUNCTIONS}

In [14] Koornwinder has given a rigorous limit transition of the continuous $q$-ultraspherical polynomials to the Jacobi functions. To recall his result we introduce the notation (cf. $[14,(1.5)]$ )

$$
R_{l}\left(e^{i \theta} ; \beta \mid q\right)=\frac{\beta^{l / 2}(q ; q)_{l}}{\left(\beta^{2} ; q\right)_{l}} C_{l}(\cos \theta ; \beta \mid q) \text {. }
$$

Koornwinder's result is (cf. [14, (1.6), but insert $-i$ in front of $(1 / 4) \mu$ on the left-hand side])

$$
\lim _{q \uparrow 1} R_{4 t / \ln q^{-1}}\left(q^{-\frac{i}{4} \mu} ; q^{\alpha+\frac{1}{2}} \mid q\right)=\varphi_{\mu}^{(\alpha, \alpha)}(t)
$$

for $\mu \in \mathbf{C}, t \in[0, \infty), \alpha \geq \frac{1}{2}$. Here $q$ must be chosen so that $4 t / \ln q^{-1} \in \mathbf{Z}_{+}$. Note that $q$ can approach 1 as $l$ approaches $\infty$. The right-hand side of (3.2) denotes a Jacobi function defined by

$$
\varphi_{\mu}^{(\alpha, \beta)}(t)={ }_{2} F_{1}\left(\begin{array}{c}
\frac{1}{2}(\alpha+\beta+1+i \mu), \frac{1}{2}(\alpha+\beta+1-i \mu) \\
\alpha+1
\end{array} ;-\sinh ^{2} t\right)
$$

(cf. $[11,(2.3)],[12, \S 2.1])$. For $\alpha=\beta, \Re(\alpha)>-\frac{1}{2}$ we have the integral representation

(3.4) $\varphi_{\mu}^{(\alpha, \alpha)}(t)=\frac{2^{\frac{1}{2}-\alpha} \Gamma(2 \alpha+1)}{\Gamma\left(\alpha+\frac{1}{2}\right) \Gamma\left(\alpha+\frac{1}{2}\right)}(\sinh 2 t)^{-2 \alpha} \int_{-t}^{t}(\cosh 2 t-\cosh 2 s)^{\alpha-\frac{1}{2}} e^{i \mu s} d s$ (cf. $[11,(2.21)],[12, \S 5.3])$.

In order to use the limit transition of the continuous $q$-ultraspherical polynomials to the Jacobi functions we first rewrite (1.6) (or (2.5)) in terms of the rescaled continuous $q$-ultraspherical polynomial $R_{l}$ (cf. (3.1)). This results in (3.5)

$$
\begin{aligned}
& \frac{\beta^{l / 2}(q ; q)_{l}}{\left(\beta^{2} ; q\right)_{l}} \sum_{n=0}^{l}{ }_{3} \varphi_{2}\left(\begin{array}{c}
q^{-n}, \beta^{2} q^{k-1}, q^{-k} \\
\beta, q^{-l}
\end{array} q, q\right) \frac{(\beta ; q)_{n}(\beta ; q)_{l-n}}{(q ; q)_{n}(q ; q)_{l-n}} e^{i(l-2 n) \theta} \\
& \quad=\frac{(\beta ; q)_{k}\left(\beta^{2} q^{l} ; q\right)_{k}}{\left(\beta^{2} ; q\right)_{2 k}} \beta^{\frac{1}{2} k} q^{\frac{1}{2} k(k-l)}\left(\beta e^{-2 i \theta} ; q\right)_{k} e^{i k \theta} R_{l-k}\left(e^{i \theta} ; \beta q^{k} \mid q\right) .
\end{aligned}
$$

In (3.5) we replace $l, \beta, e^{i \theta}$ by $4 t / \ln q^{-1}, q^{\alpha+\frac{1}{2}}, q^{-\frac{i}{4} \mu}$ as in $[14,(2.2)]$, so that the right-hand side of $(3.5)$ becomes

$$
\begin{aligned}
& \frac{\left(q^{\alpha+\frac{1}{2}} ; q\right)_{k}\left(q^{\alpha+\frac{1}{2}+\frac{1}{2} \mu} ; q\right)_{k}}{\left(q^{2 \alpha+1} ; q\right)_{2 k}} q^{\frac{1}{2} k\left(k+\alpha+\frac{1}{2}(1-i \mu)\right)}\left(q^{2 \alpha+1} e^{-4 t} ; q\right)_{k} \\
& \quad \times e^{2 t k} R_{\frac{4 l}{\ln q-1}-k}\left(q^{-\frac{i}{4} \mu} ; q^{\alpha+k+\frac{1}{2}} \mid q\right) .
\end{aligned}
$$

And because of (3.2) we see that for $q \uparrow 1$ (3.6) tends to

$$
2^{-k} \frac{\left(\alpha+\frac{1}{2}+\frac{i}{2} \mu\right)_{k}}{(\alpha+1)_{k}}(\sinh 2 t)^{k} \varphi_{\mu}^{(\alpha+k, \alpha+k)}(t)
$$

for $t \geq 0, \alpha+k \geq \frac{1}{2}, \mu \in \mathbf{C}$. 
To take the limit $q \uparrow 1$ in the left-hand side of (3.5) with the same replacements we also replace the summation parameter $n$ by $s=t-\frac{n}{2} \ln q^{-1}$. Interchanging the finite summations we obtain in a similar way as in [14, (2.3)(2.7)] the expression

$$
\begin{aligned}
& 2 \frac{1-q}{\ln q^{-1}} \frac{\Gamma_{q}(2 \alpha+1)}{\Gamma_{q}\left(\alpha+\frac{1}{2}\right) \Gamma_{q}\left(\alpha+\frac{1}{2}\right)} \frac{\left(e^{-4 t} q^{2 \alpha+1} ; q\right)_{\infty}}{\left(e^{-4 t} q ; q\right)_{\infty}} \\
& \quad \times e^{-2 t \alpha-t} \sum_{p=0}^{k} \frac{\left(q^{-k} ; q\right)_{p}\left(q^{2 \alpha+k} ; q\right)_{p} q^{p}}{(q ; q)_{p}\left(q^{\alpha+\frac{1}{2}} ; q\right)_{p}\left(e^{4 t} ; q\right)_{p}} \\
& \quad \times \frac{1}{2} \ln q^{-1} \sum_{\substack{s=-t \\
\text { step } 1 / 2 \ln q^{-1}}}^{t} \frac{\left(e^{-2(t-s)} q ; q\right)_{\infty}\left(e^{-2(t+s)} q ; q\right)_{\infty}}{\left(e^{-2(t-s)} q^{\alpha+\frac{1}{2}} ; q\right)_{\infty}\left(e^{-2(t+s)} q^{\alpha+\frac{1}{2}} ; q\right)_{\infty}} e^{i \mu s}\left(e^{2(t-s)} ; q\right)_{p}
\end{aligned}
$$

for the left-hand side of (3.5). The summation parameter $s$ in (3.8) runs through the set $\left\{-t,-t+\frac{1}{2} \ln q^{-1}, \ldots,-t+\frac{4 t}{\ln q^{-1}} \frac{1}{2} \ln q^{-1}=t\right\}$. In (3.8) we assume $t>0$ in order to avoid $\left(e^{4 t} ; q\right)_{p}=0$, at least for $1-q$ small enough. The summand in the last sum can be estimated by $e^{|\Im(\mu)| t}\left(1+e^{4 t}\right)^{k},|s| \leq t$, for $\alpha \geq \frac{1}{2}$. As in $[14, \S 2]$ we can use dominated convergence and the pointwise convergence of the $q$-binomial theorem to the binomial theorem to see that (3.8) tends to

$$
\begin{aligned}
& \frac{2^{\frac{1}{2}-\alpha} \Gamma(2 \alpha+1)}{\Gamma\left(\alpha+\frac{1}{2}\right) \Gamma\left(\alpha+\frac{1}{2}\right)}(\sinh 2 t)^{-2 \alpha} \\
& \quad \times \int_{-t}^{t}(\cosh 2 t-\cosh 2 s)^{\alpha-\frac{1}{2}} e_{2}^{i \mu s} F_{1}\left(\begin{array}{c}
-k, k+2 \alpha \\
\alpha+\frac{1}{2}
\end{array} ; \frac{e^{-2 s}-e^{-2 t}}{e^{2 t}-e^{-2 t}}\right) d s
\end{aligned}
$$

as $q \uparrow 1$. Note that we can rewrite (3.9) in operator notation if we introduce the operator $T$, which sends $\mu$ to $\mu+2 i$. Upon equating (3.9) and (3.7) we obtain as the limit result of (1.6) the following proposition.

Proposition 3.1. For $\mu \in \mathrm{C}, t>0, \alpha \geq \frac{1}{2}$ we have

$$
\begin{aligned}
{ }_{2} F_{1} & \left(\begin{array}{c}
-k, k+2 \alpha \\
\alpha+\frac{1}{2} ; \frac{T-e^{-2 t}}{2 \sinh 2 t}
\end{array}\right) \varphi_{\mu}^{(\alpha, \alpha)}(t) \\
& =2^{-k} \frac{\left(\alpha+\frac{1}{2}+\frac{i}{2} \mu\right)_{k}}{(\alpha+1)_{k}}(\sinh 2 t)^{k} \varphi_{\mu}^{(\alpha+k, \alpha+k)}(t)
\end{aligned}
$$

where $T \varphi_{\mu}^{(\alpha, \alpha)}(t)=\varphi_{\mu+2 i}^{(\alpha, \alpha)}(t)$ and $\varphi_{\mu}^{(\alpha, \alpha)}(t)$ is a Jacobi function defined by (3.3).

Note that the ${ }_{2} F_{1}$-series in (3.10) is essentially an ultraspherical polynomial $C_{k}^{\alpha}$. I thank Mizan Rahman for pointing out that (3.10) can be viewed as a projection formula for Jacobi functions.

Badertscher and Koornwinder not only proved (1.1) in their paper [4], but also a similar relation involving Jacobi functions and continuous symmetric 
Hahn polynomials instead of ultraspherical polynomials and Hahn polynomials. Explicitly (cf. [4, (6.13)] and use the symmetry $\varphi_{-\lambda}^{(\alpha, \alpha)}=\varphi_{\lambda}^{(\alpha, \alpha)}$, or the symmetry in $[4,(6.10),(5.4)]$ in $\lambda$ and $-\lambda)$,

$$
\begin{gathered}
p_{k}\left(\frac{i}{2} \frac{d}{d t} ; \frac{1}{2}\left(\alpha+\frac{1}{2}-i \lambda\right), \frac{1}{2}\left(\alpha+\frac{1}{2}+i \lambda\right)\right) \varphi_{\lambda}^{\left(\alpha,-\frac{1}{2}\right)}(t) \\
=\frac{i^{k}\left(\alpha+\frac{1}{2}+i \lambda\right)_{k}}{2^{k}(\alpha+1)_{k}}(\sinh t)^{k} \varphi_{\lambda}^{\left(\alpha+k,-\frac{1}{2}\right)}(t)
\end{gathered}
$$

for $\lambda \in \mathbf{C}, k \in \mathbf{Z}_{+}, \alpha \in \frac{1}{2} \mathbf{Z}_{+}$. Here $p_{k}(x ; a, b)$ denotes a continuous symmetric Hahn polynomial defined by

$$
p_{k}(x ; a, b)=i_{3}^{k} F_{2}\left(\begin{array}{c}
-k, k+2 a+2 b-1, a-i x \\
a+b, 2 a
\end{array}\right)
$$

(cf. references in [4]). This result is derived using the homogeneous space $S O_{0}(1, d) / S O(d)$.

Since $\varphi_{\lambda}^{\left(\alpha,-\frac{1}{2}\right)}(t)=\varphi_{2 \lambda}^{(\alpha, \alpha)}\left(\frac{t}{2}\right)$ (cf. $\left.[11,(2.8)],[12, \S 5.3]\right)$, we see that the righthand sides of (3.10) and (3.11) are essentially the same functions of $t$. This leads to the following curious proposition.

Proposition 3.2. With the notation of (3.3) and (3.12) we have

$$
\begin{aligned}
p_{k}\left(\frac{i}{2}\right. & \left.\frac{d}{d t} ; \frac{1}{2}\left(\alpha+\frac{1}{2}-i \lambda\right), \frac{1}{2}\left(\alpha+\frac{1}{2}+i \lambda\right)\right) \varphi_{\lambda}^{\left(\alpha,-\frac{1}{2}\right)}(t) \\
& =i^{k}{ }_{2} F_{1}\left(\begin{array}{c}
-k, k+2 \alpha \\
\alpha+\frac{1}{2}
\end{array} ; \frac{\tilde{T}-e^{-t}}{2 \sinh t}\right) \varphi_{\lambda}^{\left(\alpha,-\frac{1}{2}\right)}(t),
\end{aligned}
$$

for $t>0, k \in \mathbf{Z}_{+}, \lambda \in \mathbf{C}, \alpha \in \frac{1}{2} \mathbf{Z}_{+}$and $\tilde{T}$ is the operator defined by mapping $\varphi_{\lambda}^{\left(\alpha,-\frac{1}{2}\right)}$ to $\varphi_{\lambda+i}^{\left(\alpha,-\frac{1}{2}\right)}$.

Remark. For special values of the parameters continuous symmetric Hahn polynomials of differential operator argument have occurred in the works of Bateman in the 1930s. The approach by Bateman was later extended by Pasternack. The starting point of the researches of Bateman is the identity, in the notation of this paper,

$$
p_{n}\left(\frac{i}{2} \frac{d}{d t} ; \frac{1}{2}, \frac{1}{2}\right) \frac{1}{\cosh t}=\frac{i^{n}}{\cosh t} C_{n}^{1 / 2}(\tanh t)
$$

where $C_{n}^{1 / 2}$ is a Legendre polynomial. The identity (3.14) follows directly from

$$
\left(\frac{1}{2} \frac{d}{d t}+\frac{1}{2}\right)_{r} \frac{1}{\cosh t}=r ! \frac{1}{\cosh t}\left(\frac{1}{2}-\frac{1}{2} \tanh t\right)^{r} .
$$

Bateman derived for the polynomial $p_{n}(x ; 1 / 2,1 / 2)$ a generating function, orthogonality relations, integral representations, a three-term recurrence relation, difference equations, and other properties using (3.14) as the definition.

Bateman and Pasternack generalised (3.14) in several directions. Pasternack's generalisation of (3.14) also yields orthogonal polynomials, which fall in the 
class of continuous Hahn polynomials. For references to the original works and results by Bateman and Pasternack we refer to [5, Chapter V, $\S 3$, Chapter VI, $\S 8],[6, \S 4.7]$, and in particular to Nikiforov, Suslov, and Uvarov [16, §3.10.3.3], where references to related papers from the 1950s by Brafman, Touchard, Carlitz can also be found. I thank the referee for bringing the work of Bateman and Pasternack to my attention.

\section{ACKNOWLEDGMENT}

I thank Tom Koornwinder for suggesting the limit case to Jacobi functions and the referee and Mizan Rahman for their remarks concerning $§ 3$.

\section{REFERENCES}

1. R. Askey and M. E. H. Ismail, A generalization of ultraspherical polynomials, Studies in Pure Mathematics (P. Erdös, ed.), Birkhäuser, Basel, 1983, pp. 55-78.

2. R. Askey and J. Wilson, A set of orthogonal polynomials that generalize the Racah coefficients or the 6-j symbols, SIAM J. Math. Anal. 10 (1979), 1008-1016.

3. als, Mem. Amer. Math. Soc., vol. 54, no. 319, Amer. Math. Soc., Providence, RI, 1985.

4. E. Badertscher and T. H. Koornwinder, Continuous Hahn polynomials of differential operator argument and analysis on Riemannian symmetric spaces of constant curvature, Canad. J. Math. 44 (1992), 750-773.

5. T. S. Chihara, An introduction to orthogonal polynomials, Math. Appl., vol. 13, Gordon and Breach, New York, 1978.

6. A. Erdélyi, W. Magnus, F. Oberhettinger, and F. G. Tricomi, Higher transcendental functions, Vol. 1, McGraw-Hill, New York, 1953.

7. G. Gasper and M. Rahman, Basic hypergeometric series, Encyclopedia Math. Appl., vol. 35, Cambridge Univ. Press, London and New York, 1990.

8. M. E. H. Ismail and J. Wilson, Asymptotic and generating relations for the q-Jacobi and ${ }_{4} \varphi_{3}$ polynomials, J. Approx. Theory 36 (1982), 43-54.

9. H. T. Koelink, The addition formula for continuous $q$-Legendre polynomials and associated spherical elements on the $S U(2)$ qudntum group related to Askey-Wilson polynomials, SIAM J. Math. Anal. 25 (1994), 197-217.

10. Acta Appl. Math. (to appear).

11. T. H. Koornwinder, A new proof of a Paley-Wiener type theorem for the Jacobi transform, Ark. Mat. 13 (1975), 145-159.

12. __ Jacobi functions and analysis on noncompact semisimple Lie groups, Special Functions: Group Theoretical Aspects and Applications (R. A. Askey, T. H. Koornwinder, and W. Schempp, eds.), Reidel, Dordrecht, 1984, pp. 1-85.

13. __ Group theoretic interpretations of Askey's scheme of hypergeometric orthogonal poly. nomials, Orthogonal Polynomials and Their Applications (M. Alfaro, J. S. Dehesa, F. J. Marcellan, J. L. Rubio de Francia, and J. Vinuesa, eds.), Lecture Notes in Math., vol. 1329, Springer, New York, 1988, pp. 46-72.

14. _ Jacobi functions as limit cases of q-ultraspherical polynomials, J. Math. Anal. Appl. 148 (1990), 44-54.

15. __ Askey-Wilson polynomials as zonal spherical functions on the $S U(2)$ quantum group, SIAM J. Math. Anal. 24 (1993), 795-813.

16. A. F. Nikiforov, S. K. Suslov, and V. B. Uvarov, Classical orthogonal polynomials of a discrete variable, Springer Ser. Comput. Phys., Springer, New York, 1991. 
17. M. Noumi and K. Mimachi, Askey-Wilson polynomials and the quantum group $S U_{q}(2)$, Proc. Japan Acad. Ser. A 66 (1990), 146-149.

18. __ Askey-Wilson polynomials as spherical functions on $S U_{q}(2)$, Quantum Groups (P. P. Kulish, ed.), Lecture Notes in Math., vol. 1510, Springer', New York, 1992, pp. 98-103.

Department of Mathematics, University of Amsterdam, Plantage Muidergracht 24, 1018 TV AMSTERDAM, THE NETHERLANDS

E-mail address: Erik.Koelink@ris. KULeuven.ac.be

Current address: Department of Mathematics, University of Amsterdam, Plantage Muidergracht 24, 1018 TV Amsterdam, the Netherlands

E-mail address: koelink@fwi.uva.nl 